\title{
Phase transitions in dense 2-colour QCD
}

\author{
Tamer Boz, Seamus Cotter, Leonard Fister, Jon-Ivar Skullerud* \\ Department of Mathematical Physics, National University of Ireland Maynooth, Maynooth, \\ County Kildare, Ireland \\ E-mail: jonivar@thphys.nuim.ie
}

\begin{abstract}
We investigate 2-colour QCD with 2 flavours of Wilson fermion at nonzero temperature $T$ and quark chemical potential $\mu$, with a pion mass of $700 \mathrm{MeV}\left(m_{\pi} / m_{\rho}=0.8\right)$. From temperature scans at fixed $\mu$ we find that the critical temperature for the superfluid to normal transition depends only very weakly on $\mu$ above the onset chemical potential, while the deconfinement crossover temperature is clearly decreasing with $\mu$. We also present results for the Landau-gauge gluon propagator in the hot and dense medium.
\end{abstract}

31st International Symposium on Lattice Field Theory - LATTICE 2013

July 29 - August 3, 2013

Mainz, Germany

${ }^{*}$ Speaker. 


\section{Introduction}

Despite intensive theoretical efforts over the past decade and more, we do still not have a quantitative understanding of QCD at large baryon density. This is primarily due to the sign problem preventing first-principles Monte Carlo simulations in this régime. One way of circumventing this is to study QCD-like theories without a sign problem, and use these to provide a benchmark for model studies and other methods which do not suffer from the sign problem. The simplest such theory, which shares with QCD the properties of confinement and dynamical symmetry breaking, is 2-colour $\mathrm{QCD}\left(\mathrm{QC}_{2} \mathrm{D}\right)$.

In a series of papers 细, 目, 因, 㧅 we have studied $\mathrm{QC}_{2} \mathrm{D}$ with 2 flavours of Wilson fermion at nonzero baryon chemical potential $\mu$ and temperature $T$, culminating in a tentative mapping out of the phase diagram in the $(\mu, T)$ plane [3, 那. Here we will report on the determination of the phase transition lines [ $[$ ] and present new results for the gluon propagator at nonzero $\mu$ and $T$. Updated results for the equation of state are presented in a separate talk [5].

We use a standard Wilson gauge and fermion action augmented with a diquark source term to lift low-lying eigenvalues in the superfluid phase. The lattice spacing is $a=0.178(6) \mathrm{fm}$ and $m_{\pi} / m_{\rho}=0.8$, with $a m_{\pi}=0.645(8)$ [3]. We have performed simulations at four fixed temperatures, $T=47,70,94$ and $141 \mathrm{MeV}$, corresponding to $N_{\tau}=24,16,12$ and 8 respectively, for a range of chemical potentials $\mu a=0.0-0.9$. At $\mu a=0.35,0.4,0.5$ and 0.6 we have also performed temperature scans on $16^{3} \times N_{\tau}$ lattices with $N_{\tau}=4-16$. For the diquark source $j$ we have used $j a=0.02,0.04$ in order to allow an extrapolation to the physical $j=0$ limit. We refer to [3, 田 for further details about the action and parameters.

\section{Superfluid to normal transition}

Figure 1 shows the order parameter for superfluidity, the (unrenormalised) diquark condensate $\langle q q\rangle$, as a function of the temperature $T$, for $\mu a=0.35,0.4,0.5$ and 0.6. Also shown are the results of a linear extrapolation to $j=0$. We can clearly observe a transition from a superfluid phase, characterised by $\langle q q\rangle \neq 0$, at low temperature, to a normal phase with $\langle q q\rangle=0$ at high temperature, with a transition in the region $0.08 \lesssim T a \lesssim 0.12$ for all four values of $\mu$.

We have estimated the critical temperatures $T_{s}$ for the superfluid to normal transition by determining the inflection points for $\langle q q\rangle$ at $j a=0.02$ and 0.04 , and extrapolated the resulting values to $j=0$ using a linear Ansatz. The results are shown in fig. B. We see that $T_{s}$ is remarkably constant over the whole range of $\mu$-values considered. The indications are that the transition happens at a somewhat lower temperature at $\mu a=0.35$, but this point is already very close to the onset from vacuum to superfluid at $T=0, \mu_{o} a=m_{\pi} a / 2=0.32$, suggesting that $T_{s}(\mu)$ rises very rapidly from zero at $\mu=\mu_{o}$ before suddenly flattening off.

\section{Deconfinement transition}

The Polyakov loop $\langle L\rangle$ serves as the traditional order parameter for deconfinement in gauge theories, with $\langle L\rangle \neq 0$ signalling the transition to a deconfined phase. Strictly speaking, $\langle L\rangle$ is never zero in a theory with dynamical fermions, but it typically increases with temperature from 


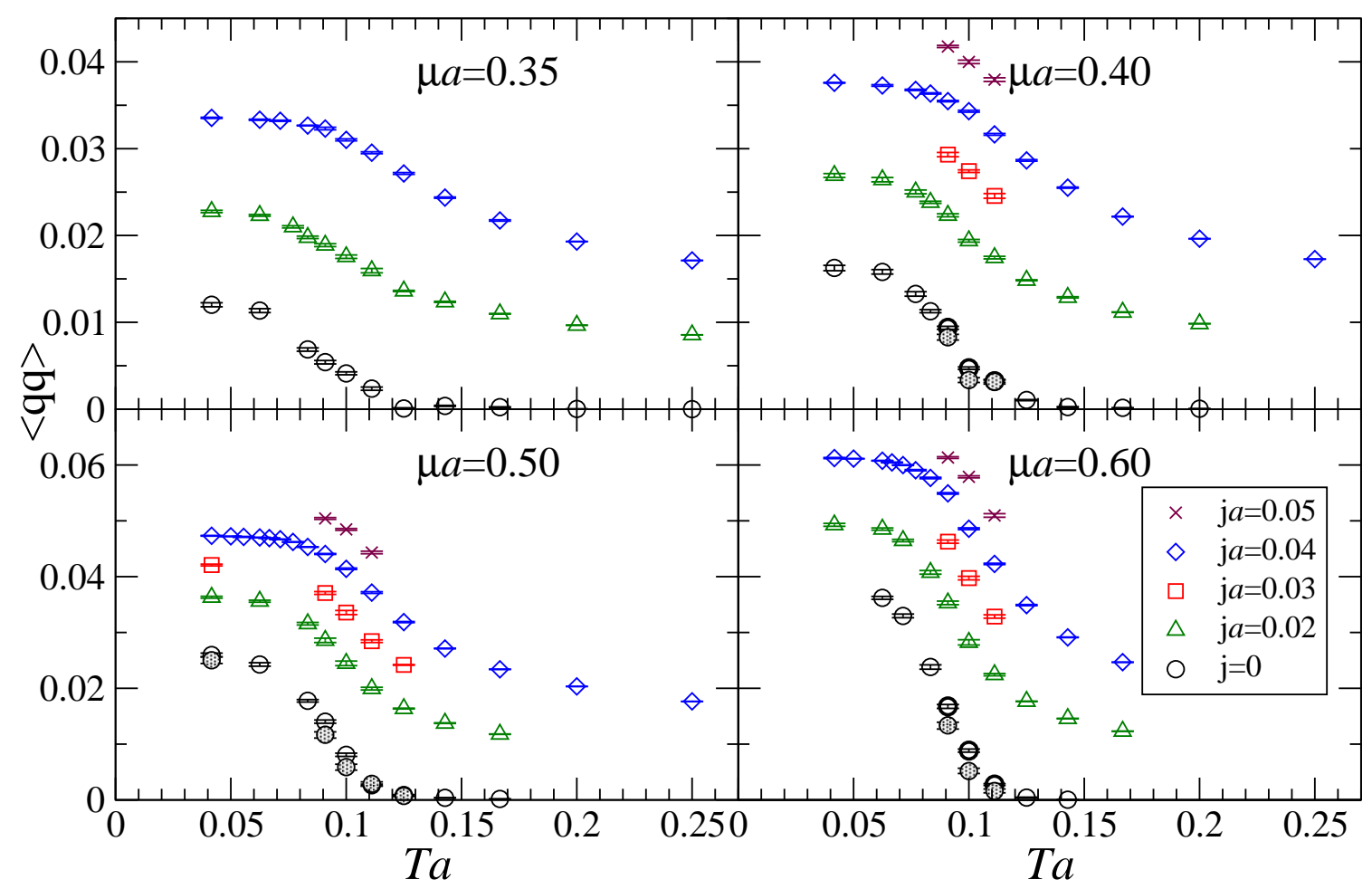

Figure 1: Diquark condensate $\langle q q\rangle$ as a function of temperature $T$ for chemical potential $\mu a=$ $0.35,0.4,0.5,0.6$ (top to bottom). The circles are data extrapolated to $j=0$ using a linear Ansatz for $j a \leq 0.04$; the shaded circles denote the results of a linear extrapolation using $j a=0.02,0.03$ only.

a very small value in a fairly narrow region, which may be identified with the deconfinement transition region. Unlike the diquark condensate, the renormalisation of the Polyakov loop depends on temperature; specifically, the relation between the bare Polyakov loop $L_{0}$ and the renormalised Polyakov loop $L_{R}$ is given by $L_{R}(T, \mu)=Z_{L}^{N_{\tau}} L_{0}\left(\left(a N_{\tau}\right)^{-1}, \mu\right)$. In order to investigate the sensitivity of our results to the renormalisation scheme, we have used two different conditions to determine the constant $Z_{L}$ 㺻, $L_{R}\left(T=T_{0}, \mu=0\right)=c$, with $T_{0}=\frac{1}{4} a^{-1}$ and $c=1$ (Scheme A) or $c=0.5$ (Scheme B). Figure 2 shows $\langle L\rangle$ evaluated in both schemes, as a function of temperature. The Scheme B data have been multiplied by 2 to ease the comparison with the Scheme A data. Also shown are cubic spline interpolations of the data and the derivative of these interpolations, with solid lines corresponding to Scheme A and dotted lines to Scheme B.

At all $\mu$, we see a transition from a low-temperature confined region to a high-temperature deconfined region. In contrast to the diquark condensate, we see a clear, systematic shift in the transition region towards lower temperatures as the chemical potential increases. For all four $\mu$ values, the Polyakov loop shows a nearly linear rise as a function of temperature in a broad region, suggesting that the transition is a smooth crossover rather than a true phase transition. This is reinforced by the difference between Scheme A and Scheme B, with the crossover occuring at higher temperatures in Scheme B. At $\mu=0$, the difference between the two schemes is small, but increases with increasing $\mu$, suggesting a broadening of the crossover.

Because of the smaller value of $Z_{L}$, our results for Scheme B are considerably less noisy than 


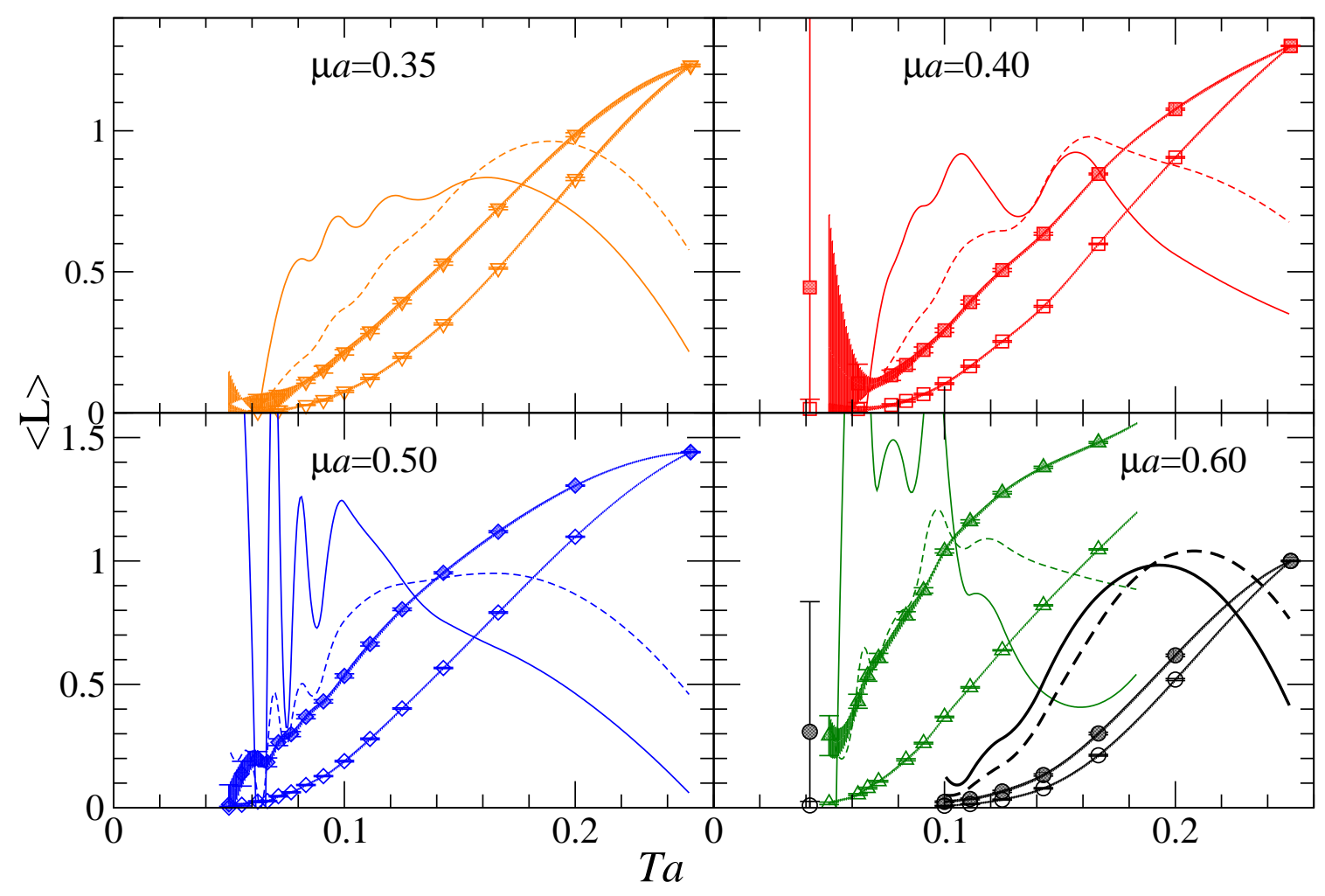

Figure 2: The renormalised Polyakov loop $\langle L\rangle$ as a function of temperature $T$ for $j a=0.04$ and $\mu a=$ $0.35,0.4,0.5,0.6$, with two different renormalisation schemes: Scheme A (solid symbols) and Scheme B (open symbols), see text for details. The solid (dashed) lines are the derivatives of cubic spline interpolations of the data points for Scheme A (B). The smaller, shaded symbols are results for $j a=0.02$. The black circles and thick lines in the bottom right panel are the $\mu=j=0$ results from [3].

those for Scheme A. For this reason, we choose to define the crossover region to be centred on the inflection point from Scheme B, with a width chosen such that it also encompasses the onset of the linear region from Scheme A.

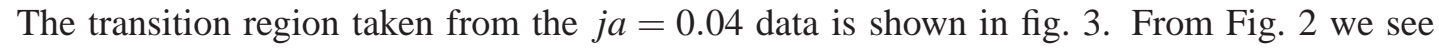
that at low $T$, the value of $\langle L\rangle$ increases as $j$ is reduced, and at $\mu a=0.6$, the crossover region will most likely move to smaller $T$ in the $j \rightarrow 0$ limit. However, we do not have sufficient statistics for $j a=0.02$ at low $T$ to make any quantitative statement about this.

\section{Gluon propagator}

One of the main motivations for studying dense $\mathrm{QC}_{2} \mathrm{D}$ on the lattice is to provide constraints on approaches which do not suffer from the sign problem. The gluon propagator provides a key input for several of these approaches, in particular functional studies using the functional renormalisation group or Dyson-Schwinger equations. These are most often carried out in the Landau gauge.

In Landau gauge only the transverse part of the vacuum propagator is non-zero. However, the external parameters break manifest Lorentz invariance, hence the gluon propagator $D$ must be 


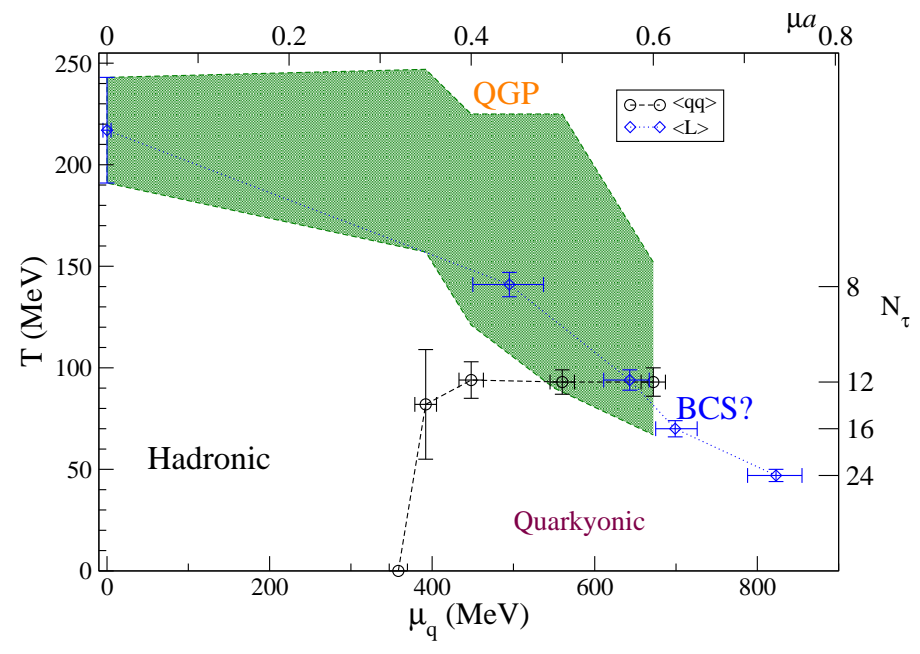

Figure 3: Phase diagram of $\mathrm{QC}_{2} \mathrm{D}$ with $m_{\pi} / m_{\rho}=0.8$. The black circles denote the superfluid to normal phase transition; the green band the deconfinement crossover. The blue diamonds are the estimates for the deconfinement line from [3].

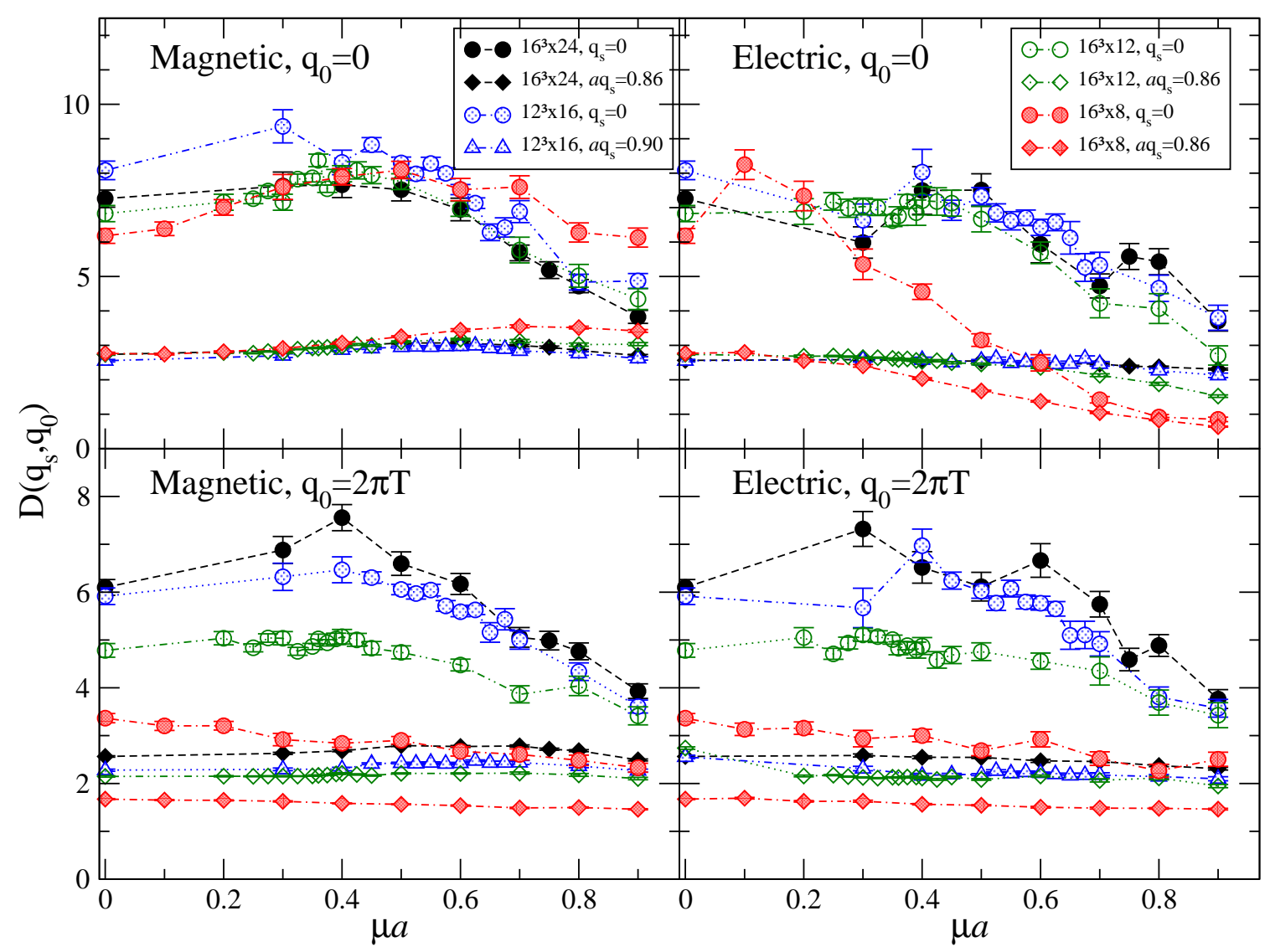

Figure 4: The zeroth (top) and first (bottom) Matsubara mode of the magnetic (left) and electric (right) gluon propagator as a function of chemical potential $\mu$ for selected values of the spatial momentum $q_{s}=|\vec{q}|$, and different temperatures. 


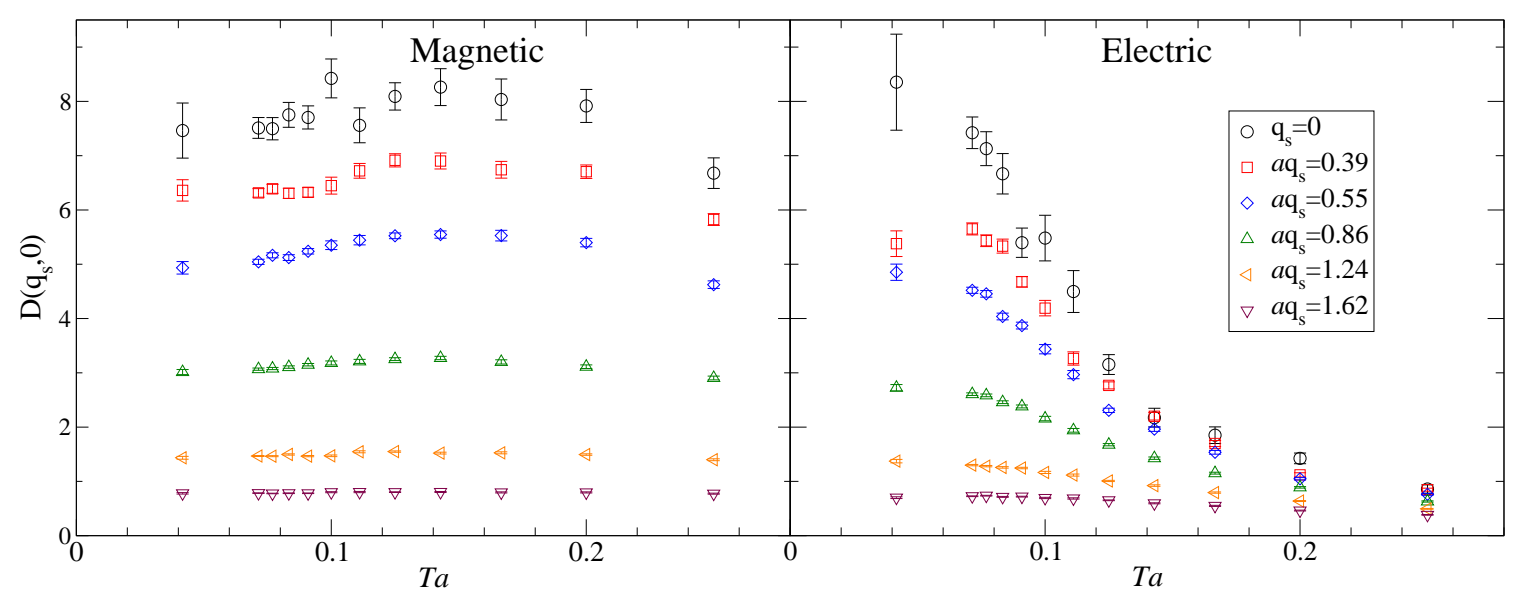

Figure 5: Thermal behaviour of the zeroth Matsubara mode of the magnetic (left) and electric (right) propagators at $\mu a=0.5$ and $j a=0.04$ on $16^{3} \times N_{\tau}$ lattices, for selected spatial momenta $q_{s}=|\vec{q}|$.

decomposed into chromoelectric and chromomagnetic modes, $D_{E}$ and $D_{M}$, respectively,

$$
D_{\mu \nu}\left(q_{0}, \vec{q}\right)=P_{\mu \nu}^{M} D_{M}\left(\vec{q}^{2}, q_{0}^{2}\right)+P_{\mu \nu}^{E} D_{E}\left(\vec{q}^{2}, q_{0}^{2}\right)
$$

The projectors on the longitudinal and transversal spatial subspaces, $P_{\mu \nu}^{E}$ and $P_{\mu \nu}^{M}$, are defined by

$$
\begin{aligned}
& P_{\mu v}^{M}\left(\vec{q}, q_{0}\right)=\left(1-\delta_{0 \mu}\right)\left(1-\delta_{0 v}\right)\left(\delta_{\mu v}-\frac{q_{\mu} q_{v}}{\vec{q}^{2}}\right), \\
& P_{\mu \nu}^{E}\left(\vec{q}, q_{0}\right)=\left(\delta_{\mu v}-\frac{q_{\mu} q_{v}}{q^{2}}\right)-P_{\mu v}^{M}\left(\vec{q}, q_{0}\right) .
\end{aligned}
$$

In this section we extend the results presented in [ [ to a wider area of the $(\mu, T)$ plane. We have fixed our gauge configurations to the minimal Landau gauge using the standard overrelaxation algorithm. The Landau gauge condition has been imposed with a precision $\left|\partial_{\mu} A_{\mu}\right|<10^{-10}$.

In figure 4 we show the two lowest Matsubara modes for selected spatial momenta as a function of chemical potential for $N_{\tau}=24,16,12,8$. The results shown are for $j a=0.04$, but we have found no significant difference for $j a=0.02$. We have investigated the volume dependence on the $N_{\tau}=24$ lattices and found it to be very mild [4]. At the three lower temperatures, both the electric and magnetic form factors are roughly independent of $\mu$ up to $\mu a \approx 0.5$, and become suppressed for large $\mu$. This changes dramatically at the highest temperature shown $\left(N_{\tau}=8\right)$, where for the lowest (static) Matsubara mode the electric form factor becomes strongly suppressed with increasing $\mu$, while the magnetic form factor for small spatial momenta has a clear enhancement at intermedate $\mu$ and an enhancement at large $\mu$ for larger spatial momenta. On closer inspection it is possible to see the onset of this behaviour also for $N_{\tau}=12$. No qualitative differences are seen between the electric and magnetic form factors for the first nonzero Matsubara mode.

We now turn to the thermal behaviour of the gluon propagator at fixed chemical potential. Fig. 5 shows the zeroth Matsubara modes of the propagators for $\mu a=0.5$ and $j a=0.04$ on $16^{3} \times N_{\tau}$ lattices as a function of temperature. The magnetic component has a very mild enhancement at 
intermediate temperatures and a slight suppression at very high $T$. In contrast, the electric propagator shows a strong suppression with increasing temperature. We note that the deconfinement crossover for this value of $\mu$ happens for $0.8 \lesssim T a \lesssim 2.0$, and that this coincides roughly with the region where the magnetic propagator is enhanced. In contrast to early studies in pure Yang-Mills theory, but in line with a recent study in QCD with twisted-mass Wilson fermions [6], there is no enhancement in the electric mode in the transition region.

\section{Summary and outlook}

We have studied the superfluid and deconfinement transition lines in $\mathrm{QC}_{2} \mathrm{D}$ in the $(\mu, T)$ plane. We find that the superfluid transition temperature is remarkably insensitive to $\mu$ for the quark mass we are using, while the deconfinement temperature is clearly decreasing as $\mu$ increases. At low temperature, the low-momentum modes of both the electric and magnetic Landau-gauge gluon propagator become suppressed relative to the (already infrared suppressed) vacuum propagator at large $\mu$, with no qualitative differences between the two form factors found. At high temperature, the static electric and magnetic propagators are found to exhibit very different behaviours, with a strong suppression of the electric form factor and an enhancement of the magnetic form factor at intermediate $\mu$. We are in the process of extending these studies to smaller quark masses as well as finer lattice spacings. In a forthcoming publication we will also study the response of the quark propagator to $\mu$ and $T$. This will enable us to directly confront the results from functional methods for these quantities.

\section{Acknowledgments}

This work has been carried out with the support of Science Foundation Ireland grant 11-RFP.1PHY3193. We acknowledge the use of the computational resources provided by the UKQCD collaboration and the DiRAC Facility jointly funded by STFC, the Large Facilities Capital Fund of BIS and Swansea University. We thank the DEISA Consortium (www.deisa.eu), funded through the EU FP7 project RI-222919, for support within the DEISA Extreme Computing Initiative. The simulation code was adapted with the help of Edinburgh Parallel Computing Centre funded by a Software Development Grant from EPSRC. We thank Pietro Giudice, Simon Hands and Jan Pawlowski for stimulating discussions and advice.

\section{References}

[1] S. Hands, S. Kim and J.-I. Skullerud, Eur. Phys. J. C48, 193 (2006), [hep-lat/0604004].

[2] S. Hands, S. Kim and J.-I. Skullerud, Phys. Rev. D81, 091502R (2010), [arXiv:1001.1682].

[3] S. Cotter, P. Giudice, S. Hands and J.-I. Skullerud, Phys.Rev. D87, 034507 (2013), [arXiv:1210.4496].

[4] T. Boz, S. Cotter, L. Fister, D. Mehta and J.-I. Skullerud, Eur.Phys.J. A49, 87 (2013), [arXiv:1303.3223].

[5] S. Cotter, P. Giudice, S. Hands and J.-I. Skullerud, PoS LAT2013 (2013), these proceedings.

[6] R. Aouane, F. Burger, E.-M. Ilgenfritz, M. Muller-Preussker and A. Sternbeck, Phys. Rev. D 87, 114502 (2013), [arXiv:1212.1102]. 